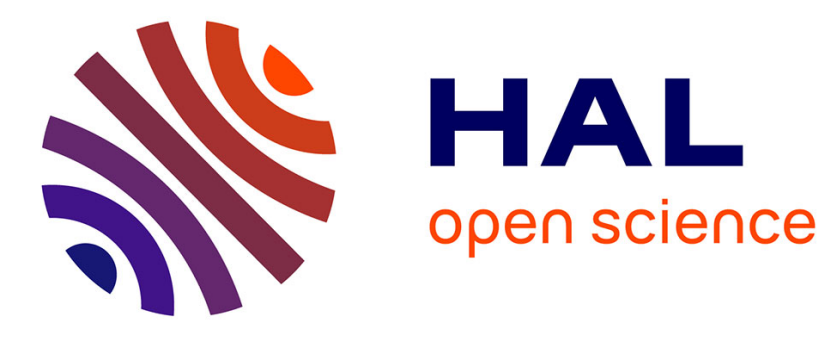

\title{
3D strain assessment in ultrasound (straus): A synthetic comparison of five tracking methodologies
}

Mathieu de Craene, Stéphanie Marchesseau, Brecht Heyde, Hang Gao, M. Alessandrini, Olivier Bernard, Gemma Piella, Antonio Porras, Lennart Tautz, Anja Hennemuth, et al.

\section{To cite this version:}

Mathieu de Craene, Stéphanie Marchesseau, Brecht Heyde, Hang Gao, M. Alessandrini, et al.. 3D strain assessment in ultrasound (straus): A synthetic comparison of five tracking methodologies. IEEE Transactions on Medical Imaging, 2013, 32 (9), pp.1632 - 1646. 10.1109/TMI.2013.2261823 . hal00840039

\section{HAL Id: hal-00840039 \\ https://hal.inria.fr/hal-00840039}

Submitted on 7 Nov 2013

HAL is a multi-disciplinary open access archive for the deposit and dissemination of scientific research documents, whether they are published or not. The documents may come from teaching and research institutions in France or abroad, or from public or private research centers.
L'archive ouverte pluridisciplinaire HAL, est destinée au dépôt et à la diffusion de documents scientifiques de niveau recherche, publiés ou non, émanant des établissements d'enseignement et de recherche français ou étrangers, des laboratoires publics ou privés. 


\title{
3D Strain Assessment in Ultrasound (Straus): A synthetic comparison of five tracking methodologies
}

\author{
M. De Craene, S. Marchesseau, B. Heyde, H. Gao, M. Alessandrini, O. Bernard, G. Piella, A.R. Porras, L. Tautz,
} A. Hennemuth, A. Prakosa, H. Liebgott, O. Somphone, P. Allain, S. Makram Ebeid, H. Delingette, M. Sermesant, J. D’hooge, E. Saloux

\begin{abstract}
This paper evaluates five 3D ultrasound tracking algorithms regarding their ability to quantify abnormal deformation in timing or amplitude. A synthetic database of B-mode image sequences modeling healthy, ischemic and dyssynchrony cases was generated for that purpose. This database is made publicly available to the community. It combines recent advances in electromechanical and ultrasound modeling. For modeling heart mechanics, the Bestel-Clement-Sorine electromechanical model was applied to a realistic geometry. For ultrasound modeling, we applied a fast simulation technique to produce realistic images on a set of scatterers moving according to the electromechanical simulation result. Tracking and strain accuracies were computed and compared for all evaluated algorithms. For tracking, all methods were estimating myocardial displacements with an error below one milimeter on the ischemic sequences. The introduction of a dilated geometry was found to have a significant impact on accuracy. Regarding strain, all methods were able to recover timing differences between segments, as well as low strain values. On all cases, radial strain was found to have a low accuracy in comparison to longitudinal and circumferential components.
\end{abstract}

Index Terms-3D Ultrasound, Validation, Tracking, Heart, Biomechanical modeling.

\section{INTRODUCTION}

Strain is an important element in the quantification of myocardial function. Although strain heavily depends on extrinsic conditions (size, preload and afterload), it decreases

M. De Craene, O. Somphone, P. Allain, and S. Makram Ebeid are with Philips Research, Medisys, Suresnes, France.

S. Marchesseau, H. Delingette, A. Prakosa, and M. Sermesant, are with the Inria-Asclepios Project, Sophia Antipolis, France.

B. Heyde, H. Gao, and J. D'hooge are with the Department Cardiovascular Sciences, Laboratory of Cardiovascular Imaging and Dynamics, Catholic University of Leuven (KU Leuven), Leuven, Belgium.

M. Alessandrini, O. Bernard, and H. Liebgott are with Université de Lyon; CREATIS; CNRS UMR5220; Inserm U1044; INSA-Lyon; Université Lyon 1; Lyon 69622, France.

G. Piella and A.R. Porras are with DTIC, Universitat Pompeu Fabra, Barcelona, Spain.

L. Tautz and A. Hennemuth are with Fraunhofer MEVIS, Bremen, Germany.

E. Saloux is with the Department of cardiology, CHU Caen, France.

The work of MEVIS was partially funded by the European Regional Development Fund (ERDF). The work of INRIA was partially funded by the Care4Me ITEA2 project. CREATIS work was realized within the frame of the US-Tagging project financed by the French National Research Agency (ANR) and the LABEX PRIMES (ANR-11-LABX-0063) within the program "Investissements d'Avenir" (ANR-11-IDEX-0007) operated by the French ANR. The work of KU Leuven was partially funded by the Research Foundation-Flanders (Belgium, FWO-Vlaanderen). UPF was supported by CDTI under the CENIT Programme (cvRemod project) and by the European Commission Seventh Framework Programme (FP7-ICT-2007-2-224495). A. R. Porras is supported by a FPU grant from the Spanish Government. when contractility is affected, as in ischemia or in cardiomyopathies [1]. Analyzing strain curves at a regional level can also reveal mechanical dyssynchrony. Strain is less sensitive to tethering from surrounding segments than velocity or displacement measurements [2], and is therefore preferred for detecting abnormal segments. Heart motion is composed of different modes including torsion, thickening across the fibers and contraction along them. Therefore, the characterization of motion and strain should be performed in 3D to avoid artifacts resulting from out-of-plane motion.

Both tagged Magnetic Resonance (MR) and echocardiography are established modalities for quantifying strain. In echocardiography, strain can be measured through velocities retrieved by Doppler tissue imaging, or displacements retrieved by tracking of the Radio Frequency (RF) signal or the B-mode envelope. Speckle tracking refers in general to tracking methods working on the B-mode envelope, extracted from the RF signal. Methods extracting motion and strain from the full RF signal are referred to as strain imaging [3], [4]. Both speckle tracking and strain imaging methods can be applied on 3D data and have the advantage of not depending on the insonification angle.

In this context, the validation of speckle tracking methods, particularly in $3 \mathrm{D}$, is a growing source of concern to ensure reproducibility of strain measurements. A recent study [5] demonstrated that inter-vendor and inter-software variability of commercially available 3D speckle tracking algorithms was high. This motivates the construction of benchmark datasets to validate 3D ultrasound (3D US) strain algorithms, with the objective of extending the work on 2D speckle tracking standardization [6] to 3D.

\section{A. Validation of $3 D$ US strain algorithms}

Several studies compared 2D and 3D US wall motion [7], global deformation [8], [9] and strain values [10], [11]. These studies compared the consistency of results, the computation time and the number of segments analyzed by $2 \mathrm{D}$ and $3 \mathrm{D}$ speckle tracking. When differences appear between 2D and 3D quantification results, these can either be explained by the intrinsic error of measuring a 3D quantity in a 2D slice [10], or by the loss of spatiotemporal resolution when going from $2 \mathrm{D}$ to $3 \mathrm{D}$.

2D tagged MR imaging is a well-validated modality for obtaining strain reference values. $2 \mathrm{D}$ speckle tracking measurements were compared to tagged MR by Amundsen et 
al. [12]. Comparing 3D speckle tracking to 2D tagged MR would lead to the same issues as when comparing $2 \mathrm{D}$ and $3 \mathrm{D}$ US measurements. In addition, myocardial tissue is imaged differently in US and MR. Hence, volume estimates obtained from the two modalities have been reported to differ significantly [13].

Because a 2D modality (being US or tagged MR) cannot be the basis of 3D US strain validation, the construction of ultrasound-specific ground truth motion and deformation data remains an open challenge. It requires 3D motion and deformation ground truth values, for which several alternatives are worth mentioning in the literature.

A first option is to simulate the US imaging pipeline. While of limited realism, the advantage is to provide data where the exact underlying motion field is known and controlled. The Field II package [14] provides a library for the calculation of pressure fields from arbitrarily shaped, apodized, and excited US transducers. It can generate the spatial impulse response specific to an US system with known characteristics. Recently, Gao et al. [15] proposed a fast alternative for generating simulated 3D US sequences in less than an hour. It accelerates the convolution of a 3D point spread function by multiple 1D convolutions while allowing the integration of various simulated or measured beam profiles as a lookup table. Elen et al. [16] applied this simulation technology to an ellipsoid model of the left ventricle (LV). For inclusion of more realistic geometries and motion, Duan et al. [17] and De Craene et al. [18] integrated a realistic electro-mechanical model in the simulation process.

As an alternative to simulated data, 3D US strain algorithms can be validated on physical phantoms, where motion and deformations can be mechanically controlled [19]. In phantoms, ground truth deformation typically relies on sonomicrometry. This technique measures at a high temporal resolution the time taken by an acoustic wave to travel between pairs of crystals. Time measurements can be converted to distances if the propagation velocity within the medium is known. However, this data is only available at few locations in the entire volume. Although the geometry of phantoms are often simplified, the obtained image quality reflects the challenges of a real US imaging system better. Polyvinyl alcohol (PVA) gels [20] have been proposed as a tissue-mimicking material for MR and US. Since the number of freeze-thaw cycles affects the properties of the material, stiffer inclusions can be embedded for quantifying the localizability of lesions, both in terms of size and stiffness extent [21].

In any case, validation on synthetic data and phantoms are preliminary to in vivo validation in animal models, being the ultimate verification step before applying image-based quantification algorithms to clinical data. For animal experiments, sonomicrometry is also taken as gold standard. It has already been used for assessing the accuracy of motion-derived measures such as twist [22] or surface area changes [23]. Segmental strain values obtained by tracking and sonomicrometry were compared by Seo et al. [24] and Heyde et al. [25]. For an extensive review of existing 3D strain techniques in echocardiography, the reader can refer to the recent review of Jasaityte et al. [26].

\section{B. Contributions of this paper}

This paper focuses on the validation of 3D US algorithms by providing publicly available synthetic images with known ground truth. A simulation pipeline including both ultrasound imaging and mechanical models is introduced. It combines a biomechanical model of the heart with a 3D ultrasound fast simulator. The biomechanical model was used to simulate two distinct pathological patterns: ischemia and dyssynchrony. For ischemia, contractility was reduced in segments related to coronary perfusion territories. For dyssynchrony, the areas of fast conduction were progressively altered for delaying activation in the lateral wall. By introducing moving scatterers in the tissue, the imaging model renders realistic 3D speckle patterns of state-of-the-art US systems. It allows to modulate the relative backscatter coefficients of tissue vs. blood pool. Pericardium was modeled as a high reflective surface with smaller motion than the myocardium.

An initial description of our validation methodology, together with a preliminary release of synthetic and phantom validation data, was presented at STACOM, a satellite workshop at MICCAI, 2012 [18]. At this workshop, different teams volunteered for processing this data and reported tracking accuracy, as compared to the provided ground truth [27], [28], [29], [30], [31], [32]. They were all invited to participate in the comparison presented in this paper, on an extended version of the STACOM database.

The five methods evaluated in this paper are tracking the speckle patterns from the B-mode images. For comparing them, both accuracy on the estimated trajectories and strain were computed using the ground truth of the mechanical model. On all datasets, tracking accuracy was quantified and compared between all methodologies. On the ischemic dataset, the ability to discriminate healthy from diseased tissue was evaluated for all techniques and strain components. On the dyssynchrony datasets, the ability to recover the delay between the strain peaks in septal and lateral walls was qualitatively evaluated.

\section{STATE OF THE ART IN 3D US STRAIN}

Dense tracking of speckle patterns from 3D US images has been approached using different methodologies. The purpose of this section is to give an overview of the different strategies existing in the literature and to put all methodologies evaluated in this paper into context. Methods evaluated in this paper are written in bold in Fig. 1.

Tracking methodologies differ by the way they establish dense correspondences and the way these correspondences are regularized. For regularization, one can distinguish between two main streams: methods adding prior information in the form of a model and methods that do not make any prior assumption on the motion field.

Prior information can be embedded in statistical or physical forms. Wang et al. [33] proposed a framework for fusing information from multiple cues such as image boundaries, signal dropouts estimates and motion statistics. Leung et al. [34] used a statistical model of cardiac motion for regularizing optical flow results. Papademetris et al. [35], [36] used a transversely 


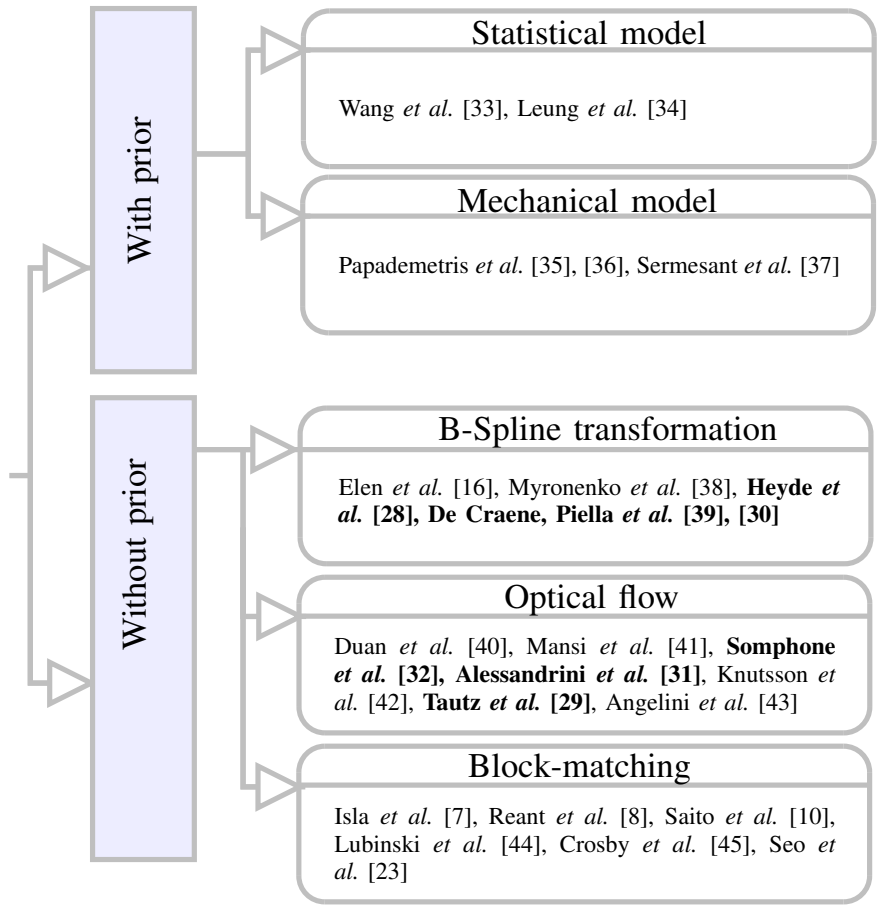

Fig. 1. Classification of motion and strain quantification methods from 3D US images. Methodologies evaluated in this paper are written in bold.

isotropic linear elastic model for regularizing shape-based tracking correspondences.

Methods that do not use prior information either perform smoothing at each iteration, or either assume that the spatial transformation can be represented by a set of generic basis functions. B-Spline methods belong to the latter category. Elen et al. [16] first applied classical Free-Form Deformation (FFD) to 3D US images in a feasibility study. The method was extended to include the time dimension in [30], [39]. The latter extend the principles of spatiotemporal registration, as developed by Ledesma-Carbayo et al. [46] in 2D US.

Since FFD typically uses a regular lattice of control points, spatial resolution in the short axis plane is low. To compensate for this, Heyde et al. [28] used an anatomical coordinate system, adapted to the left ventricular shape. Ultrasoundspecific similarity metrics, adapted to speckle statistics, were combined with FFD in [38] and [30]. A subset of optical flow-based techniques exploit the phase shift property in the frequency domain. These techniques typically combine several 1D directions through a bank of filters for computing phase differences in the Fourier domain and map them back to a displacement value in the spatial domain. Quadrature filters are used in the Morphons algorithm [42], [29]. 2D shift estimates are combined to form a 3D dense field as described in Tautz et al. [47], [29]. Spherical quadrature filters were applied in Alessandrini et al. [31] for deriving monogenic amplitude and phase. A significant advantage of the monogenic phase is its robustness regarding slight intensity changes over consecutive frames due to changes in fiber orientations or heterogeneous signal to noise ratio across the field of view.
Other tracking methods not using prior information are based on optical flow and block matching. Optical flow has been largely applied for contour tracking [40] and dense tracking [41], [32]. A review of optical flow applications in ultrasound can be found in Angelini et al. [43]. Mansi et al. [41] enforced incompressibility in the estimate of the transformation. Somphone et al. [32] replaced the standard Gaussian kernel convolution by a normalized convolution for incorporating a confidence measure in the tracking algorithm.

Block matching techniques were the first [45] to be evaluated for 3D speckle tracking in ultrasound. They directly extend the 2D speckle tracking principles. In these approaches, myocardial tissue is divided in blocks that are tracked throughout the cardiac cycle. As these methods work at a local level, an exhaustive search can be performed to find the optimal match of each block to the next frame. Spatial regularization [44] is performed after block tracking to ensure a smooth estimate of myocardial motion and deformation.

\section{Simulation PIPELINE}

Two imaging simulation technologies were combined to produce the synthetic images used in this paper. First, an electromechanical model (see Sect. III-A) was used to simulate healthy, ischemic and Left Bundle Branch Block (LBBB) conditions. Second, a distribution of scatterers was generated and moved according to the output of the electromechanical model. An ultrasound simulator took them as input to generate 3D ultrasound image sequences.

\section{A. Mechanical model}

Mechanical simulations generated for this study focused on modeling two scenarios: acute ischemia and dyssynchrony. Realistic geometries were obtained through MR segmentations of cine images (see [48] for details on the acquisition protocol). This segmentation yielded volumetric tetrahedral meshes [49]. The left ventricle was segmented from a healthy volunteer for generating the ischemic dataset. This restricts the validity of this model to a short time after infarction, at which only contractility is affected, before any structural change in the tissue would alter myocardial thickness. For dyssynchrony, the LV shape was segmented from a dilated LV geometry. The Bestel-Clement-Sorine electromechanical model described in [50], [51] was then used on this geometry for computing dense deformation through the cardiac cycle. This model was chosen for its realistic properties. It is based on energy-preserving equations, includes the Starling effect and complies with the four cardiac phases (isovolumic contraction, ejection, passive and active filling). This model showed good predictive power [52] in the context of cardiac resynchronization therapy. Moreover, tests on preload, afterload and inotropy as well as a preliminary specificity study performed in [53] proved its good physiological behavior and its ability to simulate healthy and pathological cases.

1) Acute ischemia: Several mechanical simulations were generated by modifying contractility and stiffness values regionally. Diseased areas follow the American Heart Association (AHA) 17 segments model [54] and match perfusion 
TABLE I

PARAMETER VALUES FOR ELECTROMECHANICAL SIMULATIONS (ISCHEMIC DATASET). SEE [53] FOR DETAILS. ISCH. REFERS TO MILDLY ISCHEMIC SEGMENTS SHARED BY TWO OR MORE CORONARY ARTERIES.

ISCH. ${ }^{+}$REFERS TO SEGMENTS THAT ARE CONSIDERED AS FULLY ISCHEMIC IN THE SIMULATIONS.

\begin{tabular}{llllll}
\hline Name & Units & Description & Normal & Isch. & Isch. $^{+}$ \\
\hline \hline$\sigma_{0}$ & $\mathrm{~Pa}$ & $\begin{array}{l}\text { Maximum con- } \\
\text { traction }\end{array}$ & $9 e 6$ & $1 e 6$ & $6 e 5$ \\
\hline$k_{0}$ & $\mathrm{~Pa}$ & $\begin{array}{l}\text { Maximum stiff- } \\
\text { ness }\end{array}$ & $9 e 6$ & $1 e 6$ & $6 e 5$ \\
\hline$k_{a t p}$ & $\mathrm{~s}^{-1}$ & $\begin{array}{l}\text { Contraction } \\
\text { rate }\end{array}$ & 15 & 10 & 5 \\
\hline$k_{r s}$ & $\mathrm{~s}^{-1}$ & Relaxation rate & 35 & 20 & 10 \\
\hline$c_{1}=c_{2}$ & $\mathrm{~Pa}$ & $\begin{array}{l}\text { Mooney Rivlin } \\
\text { modulus }\end{array}$ & $5 e 4$ & $1 e 5$ & $5 e 5$ \\
\hline
\end{tabular}

TABLE II

ISCHEMIC SEGMENTS FOR EACH CORONARY OCCLUSION CONSIDERED IN THE ISCHEMIC DATASET. THE ISCH. ${ }^{+}$SEGMENTS ARE SHOWN IN RED IN FIG. 2 WHILE ISCH. SEGMENTS ARE PLOTTED IN BLUE.

\begin{tabular}{lll}
\hline Case & Isch. & Isch. $^{+}$ \\
\hline LAD distal & 16 & $13,14,17$ \\
\hline LAD proximal & 16 & $8,9,13,14,17$ \\
\hline LCX & $5,11,16$ & 6,12 \\
\hline RCA & 5,11 & $3,4,10$ \\
\hline
\end{tabular}

territories of the coronary arteries. A preliminary description of the different lesion extents used in this paper can be found in [55].

A synchronous simulation was first generated by assigning a normal contractility value to all segments. Four ischemic cases were then simulated by modifying peak contractility and stiffness values in diseased segments. Two types of segments were considered for this purpose. Segments that are perfused by several coronary arteries were considered as mildly ischemic. Segments perfused by a single artery were considered as fully ischemic and had contraction parameters altered more significantly. Mechanical parameters in ischemic, mildly ischemic, and healthy segments are reported in Table I.

The first case simulated a distal occlusion of the Left Anterior Descending (LAD) artery. The second case simulated a proximal occlusion of the same artery by extending the lesion further from the apical region. Right Coronary Artery (RCA) and Left Circumflex (LCX) lesions were modeled similarly. The extent of the fully ischemic lesion for each case is shown in Fig. 2 and diseased segments are listed in Table II.

2) Dyssynchrony: Dyssynchrony, as induced by LBBB, was modeled by progressively removing areas of early activation from the LV as schematically shown in Fig. 3. This causes a progressive increase of the delay of LV with respect to the right ventricle (RV) activation, especially in the lateral wall. Therefore, the peak of the strain curves will be delayed in the lateral wall in comparison to the septum. Additionally, the early activation of the septum (through the RV) first stretches the lateral wall, before it starts contracting. This causes sign differences in septal and lateral strains in early

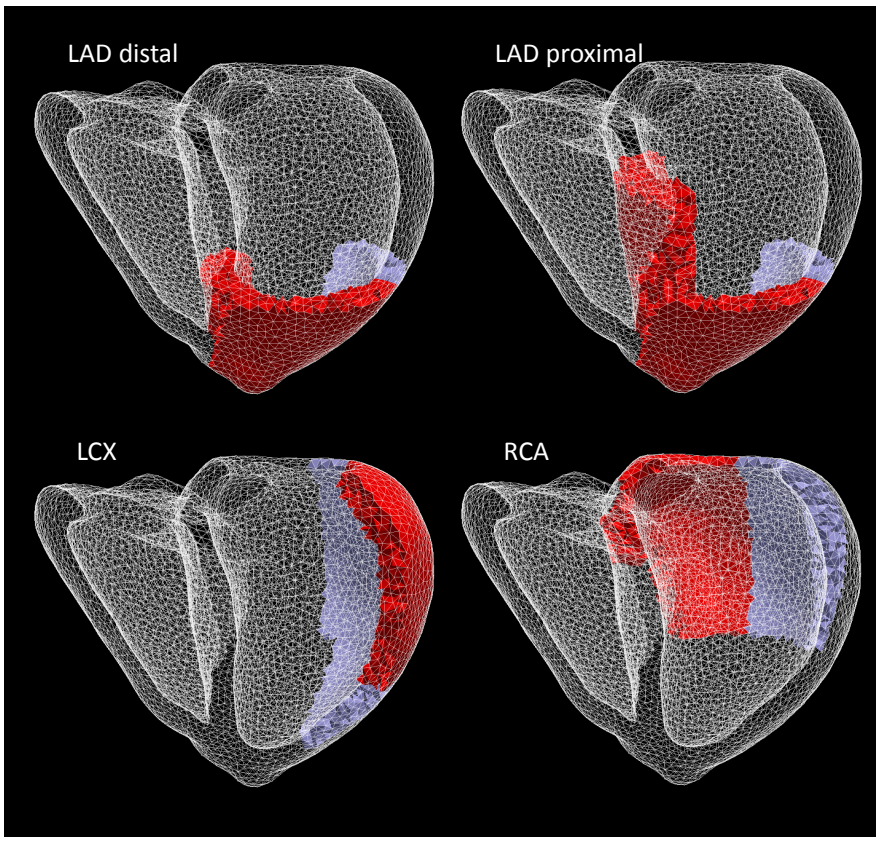

Fig. 2. Ischemic areas in four scenarios of coronary occlusion as modeled in this paper. LAD distal (top left) refers a distal occlusion of Left Anterior Descending artery, perfusing anterior segments. A proximal occlusion of that same artery will extend the lesion to septal anteroseptal segments (top right). The Left Circumflex Artery (LCX) impacts segments belonging to the lateral wall (bottom left). Finally, a Right Coronary Artery (RCA) occlusion affects inferoseptal segments (bottom right). Red segments correspond to fully ischemic segments and blue segments to mildly ischemic segments (resp. Isch $^{+}$and Isch in Tables I and II).

systole. Mechanical parameters were left unchanged from the healthy case of the ischemic dataset (Table I).

\section{B. Ultrasound model}

The ultrasound modeling process used the sequences of volumetric tetrahedral meshes returned by the electromechanical model described in Section III-A. Scattering sites were distributed within the myocardium by placing a fixed number of scatterers within each tetrahedron. The locations within one tetrahedron were taken by sampling randomly its parametric coordinates. The absolute coordinates of all scatterers were obtained from these parametric coordinates, updating the node coordinates of every tetrahedron with the result of each electromechanical simulation. As the average volume of a tetrahedron is $1.8 \mathrm{~mm}^{3}$, the average density in the myocardium is 2.2 scatterers per $\mathrm{mm}^{3}$. The blood pool was modeled as a set of randomly distributed scatterers with a reflection amplitude $\alpha$ equal to 0.5 the one in the mycoardium and a density of 0.3 per $\mathrm{mm}^{3}$.

3D B-mode image sequences were produced subsequently by introducing a fast ultrasound simulator (COLE, [15]). This approach accelerates the convolution of a 3D point spread function by performing multiple 1D convolutions (one for each line) in the spatiotemporal domain and allows for generating realistic images of specific ultrasound systems [56]. The point spread function was generated from Field II [14] in 2D and rotated to generate a $3 \mathrm{D}$ profile. 


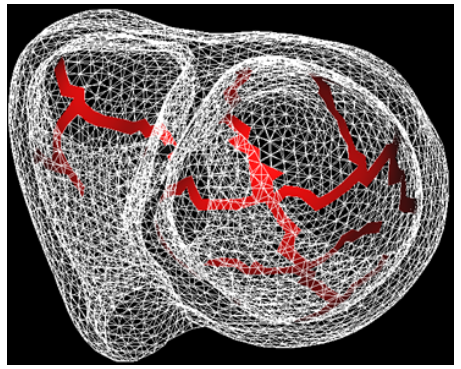

Synchronous

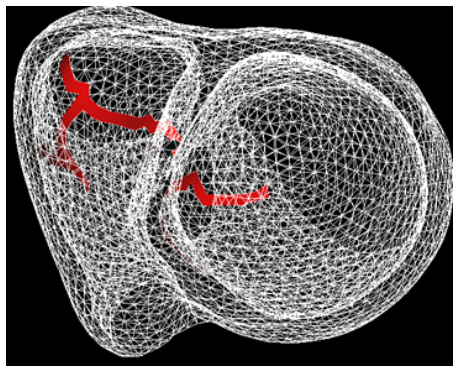

Partial LBBB

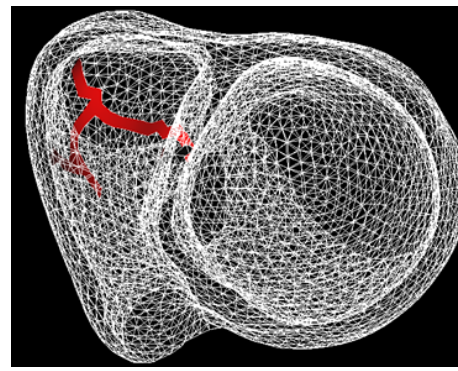

Total LBBB

Fig. 3. Electrical activation in the synchronous case, partial and total LBBB. The red stripes show the areas of early activation at the endocardium. All triangles belonging to the $\mathrm{LV}$ and the septum were progressively removed for simulating LBBB.

In the present study, the US system was sampling at 50 $\mathrm{MHz}$ and equipped with a phased array transducer, which was centered at $3.3 \mathrm{MHz}$ and transmitting a Gaussian pulse with a $-6 \mathrm{~dB}$ relative bandwidth of $65 \%$. A symmetric transverse two-way beam profile was assumed, focusing at $80 \mathrm{~mm}$ when transmitting and dynamically focusing on receive. The simulated images consisted of $107 \times 80$ lines in azimuth and elevation direction over an angle of $80 \times 80$ degrees, resulting in a frame rate of $30 \mathrm{~Hz}$ due to the use of parallel beam forming. After mapping to a Cartesian coordinate system, the final data sets consisted of $500 \times 400 \times 500$ isotropic voxels with a voxel size of $0.46 \mathrm{~mm}$. More details on the ultrasound model can be found in Gao et al. [15].

We generated two versions of the ischemic dataset to study the impact of having a very intense pericardium in the vicinity of myocardial tissue. For generating this layer, the epicardium was extracted and scaled by a factor of 1.2 from the LV center point. This surface was then moved using the displacements of the epicardium scaled down by a factor of two. Scatterers were placed on this surface in a similar way as in the myocardium. The reflection amplitude of these scatterers was set to three times the one in the myocardium. Additionally, the images with pericardium had a reduced field of view compared the ones without pericardium. The aperture of the ultrasound cone was increased from $60^{\circ}$ (with pericardium) to $80^{\circ}$ (without pericardium). This was designed for studying the impact of two typical artifacts in 3D ultrasound images: the presence of a bright pericardium moving at a different speed than the myocardium and a reduced field of view. On the dilated version, a single version with pericardium was generated. As most of the pericardium fell outside the field of view, it was expected to have less impact than on the normal geometry. An example of generated synthetic image is shown in Fig. 4 for the normal geometry, with and without pericardium.

For the normal case, we further modified the relative reflection amplitude $\alpha$ between the myocardium and the blood pool to $0.75,1$ and 1.5 to cover different Signal to Noise Ratios (SNRs). This preserves the speckle pattern within the tissue but alters the contrast between myocardium and blood pool. Hence, at low SNRs, tracking cannot rely anymore on propagating deformations estimated from contours. This can be useful for assessing the ability of each evaluated methodology to track texture rather than edges. Fig. 5 plots one image slice for the $4 \alpha$ values considered in this paper.

\section{EVALUATED TRACKING METHODOLOGIES}

This section briefly describes the five methodologies under comparison in this paper. These methods were subjected to a preliminary evaluation in the STACOM 2012 workshop. Complete details on each methodology can be found in the proceedings of this workshop [28], [30], [32], [31], [29]. For self-completeness, each method is here briefly described and will be labeled in the remainder of this paper by the institution that contributed its implementation.

\section{A. Creatis}

Creatis contributed a monogenic phase-based tracking algorithm. Their algorithm is an extension to 3D of the one proposed by the same authors in Alessandrini et al. [57]. In this scheme, the monogenic phase is obtained by the response of image intensities to four 3D spherical quadrature filters. The traditional assumption of intensity conservation is then replaced by assuming phase conservation for all material points. The transformation is assumed to be locally affine. B-Spline windows are defined over the image domain across multiple scales. A coarse to fine approach is used to iteratively refine motion estimates and account for large deformations. Apart from the B-spline functions used for computing the window sizes, this method did not perform any global regularization. Further details about this method are provided in Alessandrini et al. [31], [57]. The algorithm was implemented in Matlab. The processing time for the entire sequence corresponding to the normal simulation was about 39 minutes and 45 seconds on a $3.47 \mathrm{GHz}$ processor (64 bits, 6 cores).

\section{B. Philips}

Philips provided a fast implementation of a demons-like algorithm. Samples are collected over the myocardial domain and tracked iteratively along the sequence. The displacement field is obtained by convolution of Dirac functions placed at each sample with a Gaussian kernel. Optical flow is computed backward and forward through the sequence, in a coarse-tofine sampling of image intensities. Further details are provided in Somphone et al. [32]. The algorithm was implemented in $\mathrm{C}++$. The processing time for an entire sequence (normal case) was 45 seconds on a $2.5 \mathrm{GHz}$ processor (64 bits, 4 cores). 

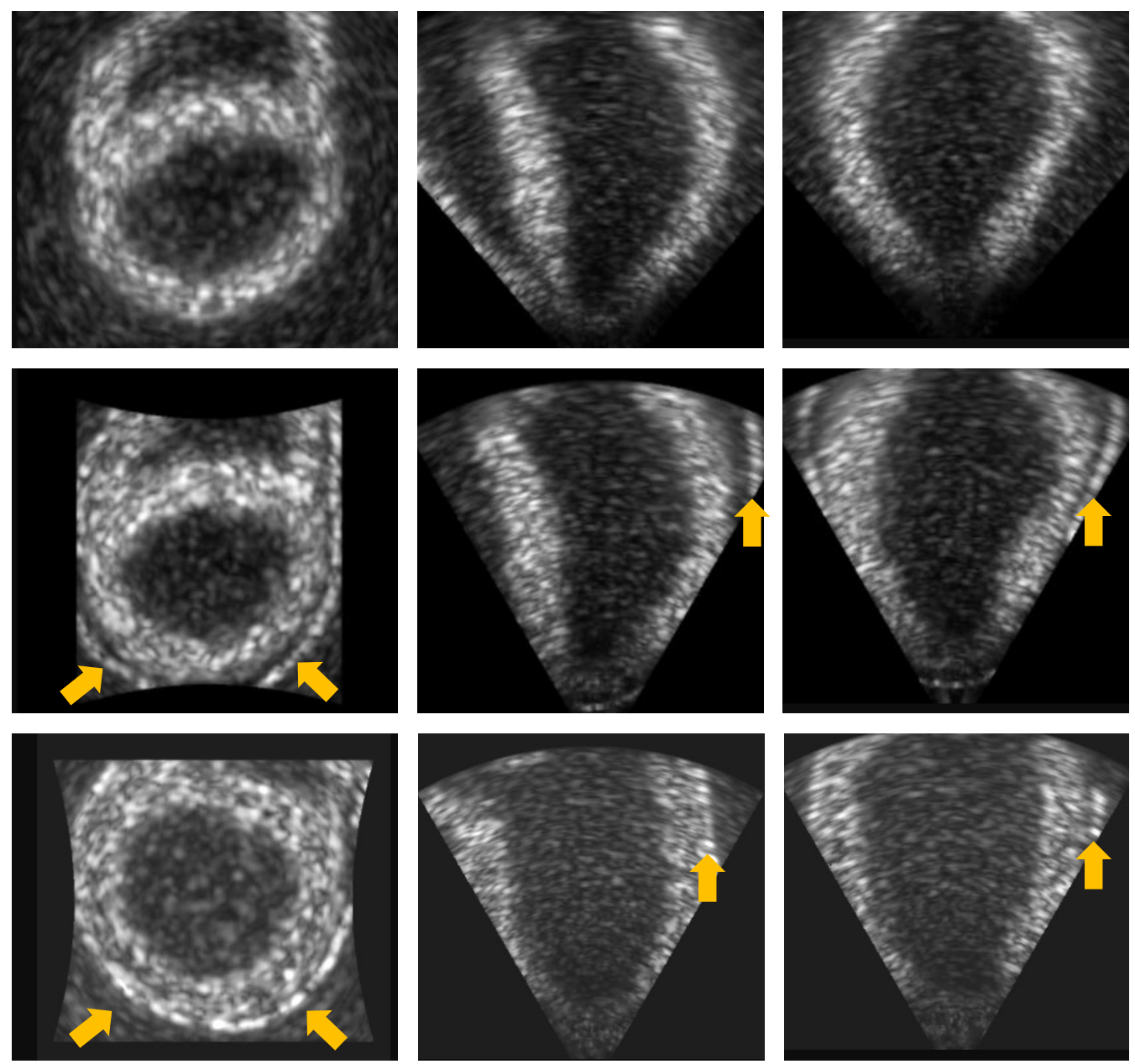

Fig. 4. 3D synthetic cardiac ultrasound images with (middle and bottom) and without (top) pericardium (as indicated by arrows) for the normal geometry. The images with pericardium had a smaller aperture to study the impact of a reduced field of view. The bottom line shows images obtained from the dilated geometry, with pericardium.

\section{KU Leuven}

KU Leuven recently proposed an extension of the FFD model [58] adapted to the LV shape. In this scheme, Bspline basis functions are locally oriented in the physiologically relevant directions of the endocardium, i.e. the radial, longitudinal and circumferential direction, as opposed to the definition on a cubic lattice in the original FFD formulation. Motion estimation can then be expressed in an anatomically equivalent space by image intensity interpolation where standard FFD registration applies. After tracking, trajectories are mapped back to cartesian space for displacement and strain computations. The reader can refer to Heyde et al. [28] for further explanations. The algorithm was implemented in $\mathrm{C}++$. It takes approximately 120 mins to process a full sequence on a 4-core $2.8 \mathrm{GHz}$ laptop processor with $8 \mathrm{~GB}$ RAM.

\section{D. $U P F$}

UPF provided another extension of the FFD algorithm to handle the temporal dimension. A non-stationary velocity field is estimated by processing the entire image sequence at once. For this paper, the similarity metric was developed to combine sequential and fixed-reference terms. The sequential term was adapted to speckle statistics for capturing local deformation, while the fixed-reference term avoids drift. A detailed description of this algorithm can be found in Piella et al. [30]. The algorithm was implemented in $\mathrm{C}++$. The computation was about 8 hours on quad-core Intel Xeon Linux server (2.66 GHz CPU, 16 GB RAM).

\section{E. MEVIS}

MEVIS contributed an implementation of the Morphons algorithm originally proposed by Knutsson et al. [42]. This techniques estimates local spatial shifts through quadrature phase differences. A confidence map is iteratively estimated and refined for accumulating iterative displacement estimates. As the method was originally $2 \mathrm{D}$, it is extended to $3 \mathrm{D}$ datasets by sampling the sequence in a sparse set of long axis and short axis planes. Displacement estimates are combined to form the final 3D motion field by summing contributions 

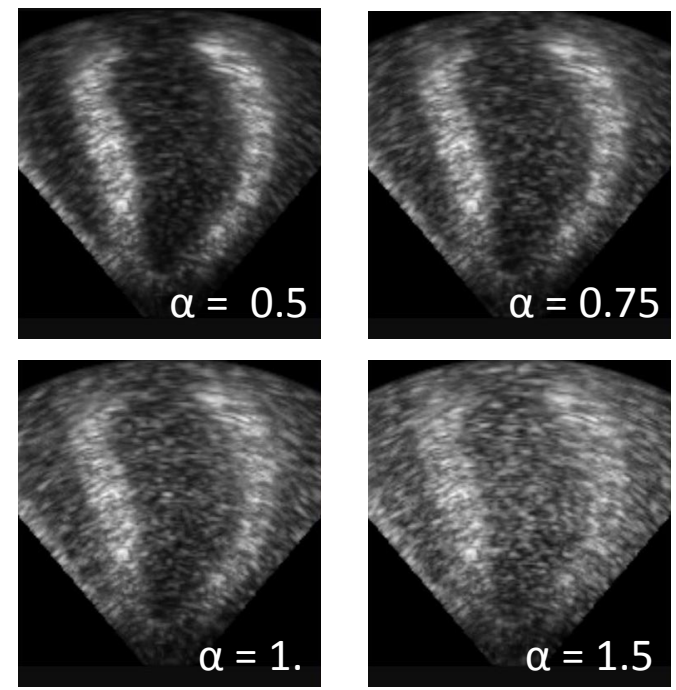

Fig. 5. Ultrasound images for decreasing contrasts between tissue and blood pool. The coefficient $\alpha$ next to each image shows the relative amplitude of tissue with respect to blood pool scatterers.

from the different planes, as described in Tautz et al. [29]. The algorithm was implemented in the MeVisLab environment (C++/Python). The computation time per case (full sequence, normal simulation) was about 90 minutes on a 8 -core $3 \mathrm{GHz}$ processor with $8 \mathrm{~GB}$ RAM.

\section{F. Timing and practical organization of the comparison}

All participants involved in this study were asked to submit a first version of their tracking results at the time of the STACOM 2012 workshop. A preliminary comparison and discussion of all methods was performed at this workshop on a previous set of simulated ultrasound images [18]. After receiving a first feedback on the performance of their method, each participant was offered the possibility to update his results two months after the workshop. Philips, MEVIS and Creatis sent an update of their results while UPF and KU Leuven maintained their original submission.

\section{GENERATED DATA AND GROUND TRUTH DEFINITION}

A total of 13 image sequences were generated for this paper. The data was divided in three groups. The first group includes the ischemic simulation with pericardium in which the normal case (no lesion) and the four lesions shown in Fig. 2 are included. The second group used the same mechanical simulations but included pericardium in the ultrasound simulation. Finally, the third group contained three sequences with the dilated geometry corresponding to the three activation patterns shown in Fig. 3.

The sequence of volumetric meshes, as obtained from the mechanical simulation, defines the ground truth. All points out of the field of view were excluded from the analysis. Ground truth strain values were obtained by approximating the transformation within each tetrahedron as an affine transform $F$. Strain was derived from the $F$ matrix using classical Cauchy formulation

$$
\epsilon=\frac{1}{2}\left(F F^{t}\right)-I
$$

It was later projected on the local cardiac coordinate system of longitudinal $l$, radial $r$ and circumferential $c$ directions according to

$$
\epsilon_{d}=d^{t} \epsilon d \text { with } d \in\{r, l, c\} .
$$

The reader can refer to the appendix in Petitjean et al. [59] for justifications of these strain definitions.

The following data is available from http://bit.ly/3dstraus as supplemental material for this paper:

1) Generated $3 D$ ultrasound images

2) Ground truth volumetric meshes with local cardiac coordinate system

3) Simple code for loading meshes and computing ground truth or tracking-based radial, circumferential and longitudinal strains

4) The output of each evaluated methodology described in Section 5 as a sequence of volumetric meshes (obtained by propagating the first mesh using the tracking result)

\section{RESUlTS}

Accuracy on motion and strain were evaluated separately for all methodologies. First, tracking accuracy was analyzed by quantifying errors on the displacement field. Second, strain accuracy was qualitatively assessed on bull's eye plots and temporal strain curves were evaluated per segment. Bias and standard deviation on strain errors were also computed for all datasets.

\section{A. Motion accuracy}

Global accuracy was computed by concatenating displacement errors for all nodes of the LV volumetric mesh at all time points and for all datasets within a group. The three groups considered here were defined in Section V: ischemic without pericardium (5 sequences), ischemic with pericardium (5 sequences) and dyssynchrony (3 sequences). The 3 sequences with low SNRs were excluded from this analysis. The resulting distributions are plotted in Fig. 6 per methodology in each group. Overall, the dyssynchrony dataset gave higher errors than the ischemic one. This cannot be attributed to image quality since ultrasound simulation parameters were kept identical in the two groups. The dilated geometry was intentionally cropped in the lateral walls by the field of view. This mimics the well-known difficulty for the sonographer of adjusting the field of view to cover the largest part of the LV. Indeed, increasing the field of view to more scanning lines would be at the expense of a decrease in temporal resolution. As visible from Fig. 6, the difficulty of epicardial points entering and leaving the field of view alters significantly the accuracy of all evaluated algorithms. On the data without pericardium, KU Leuven obtained the highest accuracy, closely followed by Philips and Creatis. UPF and MEVIS were next, obtaining median accuracy on trajectories close to $1 \mathrm{~mm}$. Among these, Creatis and UPF showed the highest maximum error, indicating the presence of some outliers. Similar trends were observed on ischemic without pericardium and dyssynchrony datasets. The presence of pericardium increased the number of outliers for 
all methodologies, whereas median error was kept relatively stable. This likely relates to the fact that all methods (except Creatis) defined a mask around the myocardium that preserved overall robustness of the tracking result.

To refine this analysis, two additional plots per methodology are provided in Figures 7 and 8. First, end-systolic errors are plotted as color maps (Fig. 7). Interestingly, regions of higher errors vary from one methodology to another. For all methods, basal segments were prone to tracking error. This could be the result of the decreasing spatial resolution with increasing depth (i.e. distance from the transducer) due to the diverging image lines. Second, error distributions were plotted over time for all methodologies (blue continuous curve and box plots in Fig. 8). As expected, errors were monotically increasing for all methodologies during systole. Average error over late diastole (end of the cycle) was higher than over systole (shown as a vertical bar in Fig. 8). Also, for most methods, error dispersion tended to be higher towards the end of the cycle. This might indicate some residual drift in the estimated trajectories. We then looked at the impact of a loss of contrast between the myocardium and the blood pool, quantifying the image sequences generated from the normal simulation at different SNR levels as described in Sect. III-B. Fig. 8 plots median accuracy curves from the highest SNR level (in blue) to the lowest (in red). For most methodologies, the lowest contrast lead to a significant accuracy loss and median error exceeded on average the $3^{\text {rd }}$ quartile limits from the high SNR error distribution. This increase was more moderated for UPF and Philips. In the case of UPF, this is likely related to the increased robustness obtained by solving for the deformation from the entire sequence. In the case of Philips, only points belonging to the mycoardium in the first frame were tracked. Hence, image gradient and Demonsbased forces were only affected at points in the vicinity of the myocardial borders.

\section{B. Strain accuracy}

We first quantified the average and standard deviation of segmental strain errors for each component, considering all time points and datasets. These measures are reported in Table III. In general, the accuracy of radial strain was found to be lower than circumferential and longitudinal components. For longitudinal strain, the levels of standard deviation are around $2 \%$ or more. Since the peak value of longitudinal strain was about $5 \%$ on the synchronous case, these levels of errors are too high for discriminating healthy from diseased segments using longitudinal strain. This is mainly due to a limitation of the synthetic dataset, as discussed in Sect. VII. All methods were found to have a standard deviation of about $2 \%$ or less for circumferential strain. These levels of accuracy can be considered as sufficiently high for discriminating ischemic segments. Indeed, in our simulations, ischemic segments had circumferential strains reduced over $10 \%$ at the end of systole.

The analysis was then focused at end of systole by generating Bland-Altman plots, using all datasets (except the ones at low SNRs) and including the estimated strain per segment. Results for circumferential strain are plotted in Fig. 9. Since
TABLE III

STRAIN ACCURACY REPORTED FOR ALL CASES. BOTH MEAN ERROR AND STANDARD DEVIATION ARE REPORTED FOR ALL TIME POINTS.

\begin{tabular}{lrrrrrr}
\hline & \multicolumn{2}{c}{ RADIAL } & \multicolumn{2}{c}{ CIRC. } & \multicolumn{2}{c}{ LONG. } \\
\cline { 2 - 7 } & Bias & Std. & Bias & Std. & Bias & $\begin{array}{c}\text { Std. } \\
(\%)\end{array}$ \\
& $(\%)$ & $(\%)$ & $(\%)$ & $(\%)$ & $(\%)$ \\
\hline KU Leuven & -3.5 & 6.7 & 0.2 & 1.1 & -0.1 & 1.8 \\
\hline Creatis & -4.4 & 9.2 & 0.4 & 1.7 & 0.3 & 2.3 \\
\hline Philips & -2.8 & 10.2 & -0.2 & 1.5 & 0.2 & 2.5 \\
\hline UPF & 1.6 & 8.9 & 0.6 & 2.1 & 1.2 & 5.6 \\
\hline MEVIS & -4.3 & 8.3 & 0.3 & 2.2 & 0.4 & 3.1 \\
\hline
\end{tabular}

ground truth is available here, we plot the measurement error against the true value. Fig. 9 shows that no clear relationship exists between the quantity to be estimated and the measurement error. Bias and limits of agreements (defined as two times the standard deviation) are plotted as horizontal dashed lines.

For analyzing the spatial distribution of strain, and compare it to the ground truth, end-systolic strain "bull's eye" maps were generated for all strain components. Fig. 10 plots the resulting maps for the normal case. Maps for one ischemic (LCX) case are shown in Fig. 11. Segments corresponding to the lesion were highlighted in bold in Fig. 11. By comparing Fig. 10 and 11, it appears that ischemic segments are well discriminated using circumferential strain. This was not the case for the longitudinal component due to the low values of end-systolic longitudinal strains. Radial strain values were consistently lower in the diseased segments. However, when comparing radial values to the ground truth, it appears that most of the challengers (except UPF), underestimated radial strain. This is likely due to excessive spatial smoothing, that tends to reduce peak deformation in the radial direction.

Fig. 12 plots strain curves obtained from the "Total LBBB" case (see Fig. 3) for all components in two opposed segments at mid-level: \#9 (inferoseptal) and \#12 (anterolateral). The purpose was to determine if timing differences were qualitatively well recovered between dyssynchronous walls. In the radial direction, due to time inconsistencies (Philips) or to potentially excessive regularization (MEVIS, Creatis), time-to-peak values were not accurate enough for quantifying dyssynchrony. Accuracy on circumferential and longitudinal components was sufficiently high for qualitatively observing delays between septal and lateral strain curves. On this dataset, UPF and KU Leuven show the highest accuracy on strain curves, with a very limited underestimation and shift of strain peaks in comparison with others.

Finally, the impact of a decreasing contrast between blood pool and tissue on strain accuracy was assessed for all methodologies using the 4 sequences shown in Fig. 5. The differences between true and estimated circumferential strains are plotted as box plots in Fig. 13 for decreasing SNR levels. All methods increased the spread of strain errors at lower contrasts. Overall, median strain accuracy was preserved, except for MEVIS and Creatis that appeared more sensitive than other methodologies. KU Leuven had the highest accuracy on circumferential strain at the lowest SNR, followed by Philips. It should be noted 


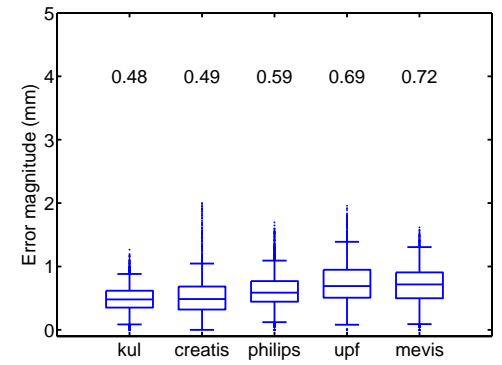

Isch. no pericardium

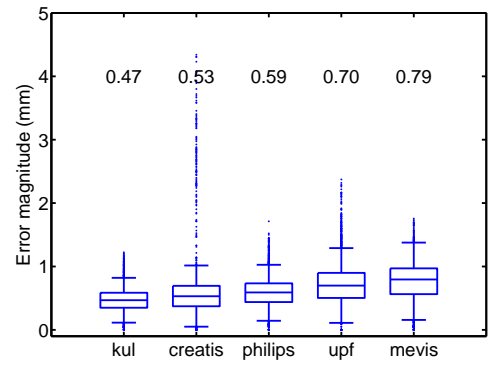

Isch. with pericardium

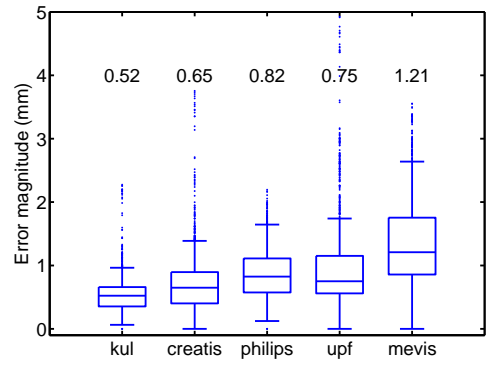

Dyssynchrony

Fig. 6. Global accuracy (on the displacement field) at all time points reported in three sub-groups: ischemic without pericardium, ischemic with pericardium and dyssynchrony.
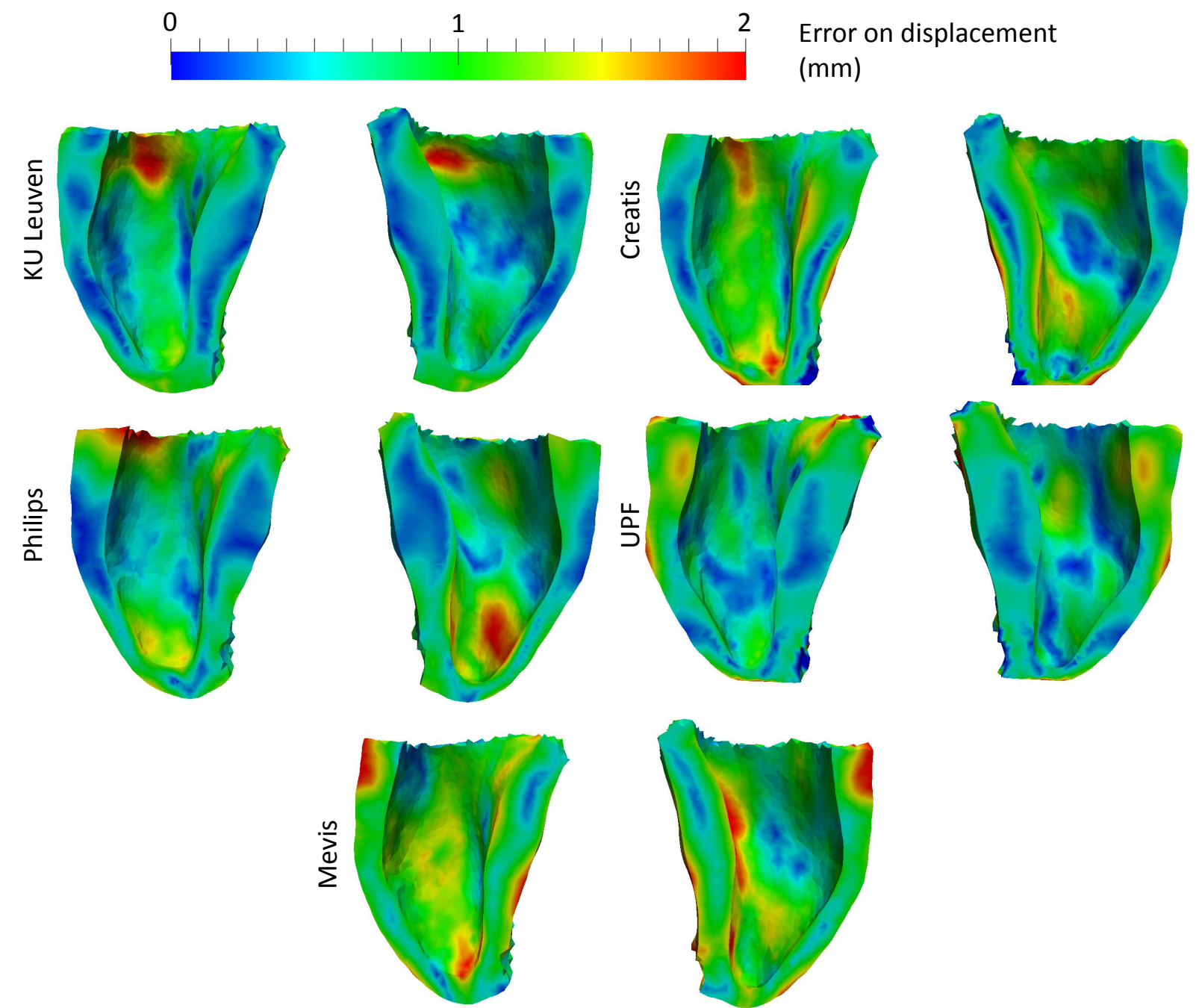

Fig. 7. Tracking accuracy plotted over space at end of systole for all challengers (normal synchronous case - no pericardium). The LV meshes are cut in two along a plane perpendicular to the septum and the lateral wall, showing the anterior LV on the left and the inferior LV on the right. 

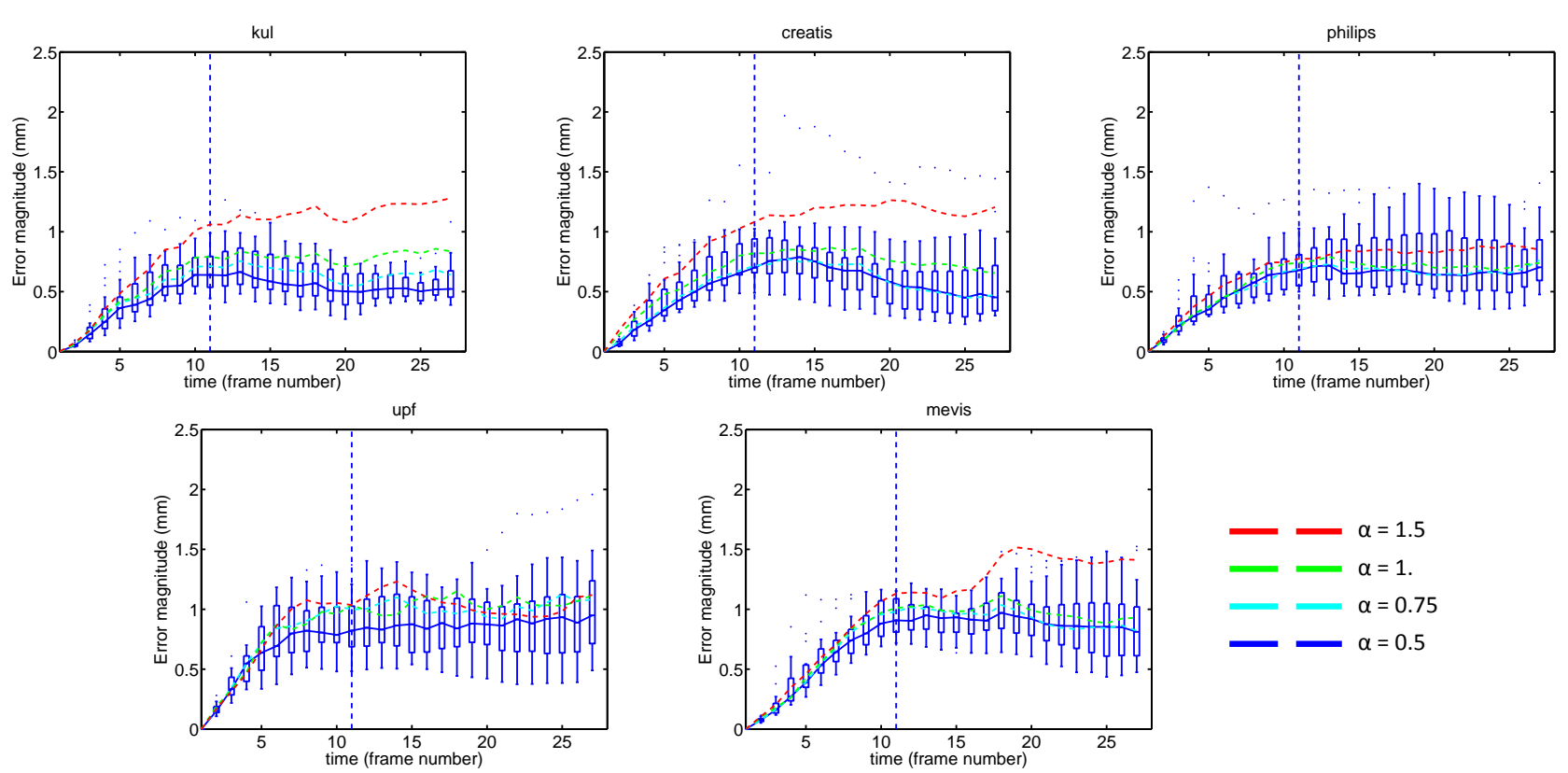

Fig. 8. These plots illustrate how accuracy evolves for each methodolgy over time and different SNRs between myocardium and blood pool intensities. Blue continuous curves and box plots correspond to the normal case without pericardium at the highest SNR. The dashed curves show the evolution of median accuracy when SNR decreases. The lowest SNR level is plotted in red. See the legend for intermediate SNR colors and Fig. 5 for the meaning of $\alpha$. The dashed vertical bar indicates the end of systole.
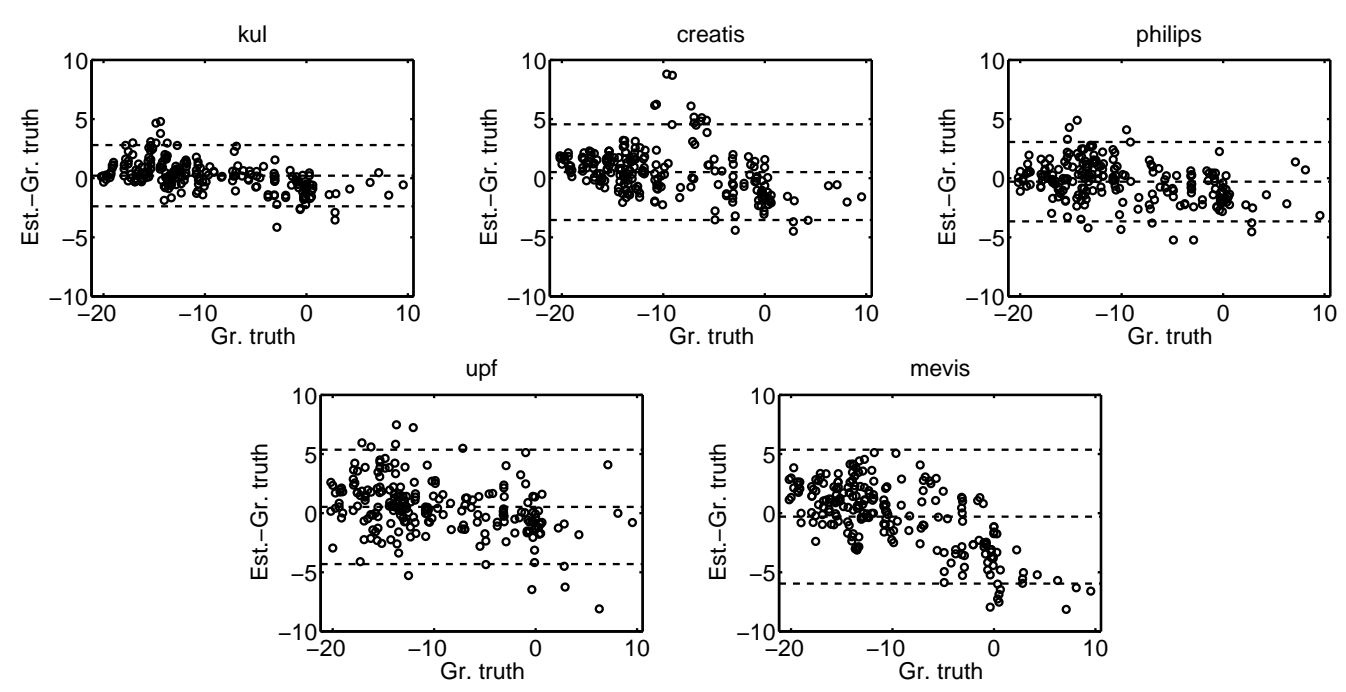

Fig. 9. End-systolic Bland-Altman plots of circumferential strain estimates for each methodology. Horizontal dashed bars indicate the limits of two standard deviations around the mean. The central horizontal dashed bar shows the bias (mean difference between true and estimated values) for each methodology. Values are averaged per segment for all datasets, yielding $17 \times 3$ values in each plot.

that, even at the lowest SNR, the accuracy on circumferential strain (ranging from 1 to $5 \%$ ) is still sufficient for detecting the ischemic segments as simulated in Sect. III-A, since the reduction in peak systolic strain exceeds $15 \%$ in pathological segments.

\section{DISCUSSION}

The main purpose of this paper is to introduce an open database for the benchmarking of 3D strain algorithms in ultrasound. All results and conclusions presented here are only valid for this particular series of generated datasets. A thorough validation needs to include experiments on phantom data and patients, although we believe synthetic data is a necessary first step. The general framework for validation that we aim to build progressively is described in [18].

The synthetic data generated for this paper suffers from the following limitations. First, the ultrasound image reconstruction around the apex yielded a low contrast between tissue and blood pool. Although image quality is typically low around the apex due to near field artifacts, the appearance of this artifact is usually different from what was obtained in these simulations. Moreover, clutter noise typically makes tracking and segmentation challenging, particularly towards the apex, but this was not included in the current simulation model. Ad- 

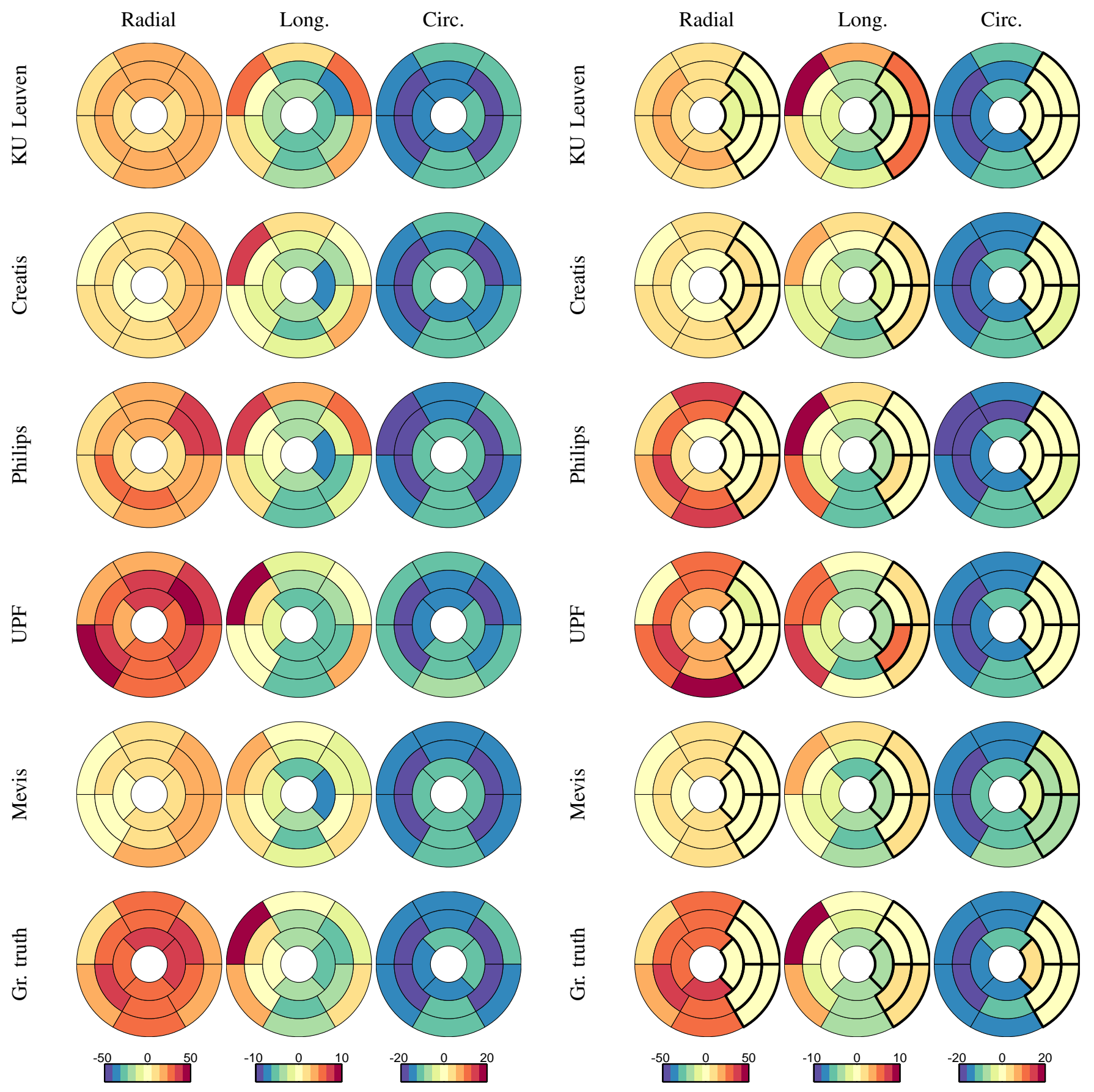

Fig. 10. End-systolic strains for the normal synchronous case. Ground truth and tracking results (5 methods) are shown on the endocardium in a bull's eye representation.

ditionally, pericardial motion was modeled in a simplistic way. More realistic approaches could be including real images for learning ultrasound artifacts [60], [61] and incorporating nonlinear propagation effects [62]. Second, regarding mechanical deformation patterns, several improvements could be made towards more realistic simulations. Longitudinal strain was found to have low values with respect to normal deformation ranges reported in the literature [63]. Additionally, the endsystolic LV shape was close to an ellipse, whereas it should remain globally circular for a normal contraction pattern.
Fig. 11. End-systolic strains for the ischemic case (LCX territory). Ground truth and tracking results (5 methods) are shown on the endocardium in a bull's eye representation. Ischemic segments are outlined in bold.

Further effort in personalizing the fiber distribution and the pressure constraints could help to improve the realism of the mechanical simulations. The apex was kept fixed for the ischemic dataset. This induced zero deformation in that area and annihilated torsion. Boundary conditions should be only set using tangential constraints on the pericardium. This was done for the dyssynchrony dataset, to allow for apical rocking. It needs to be generalized to all mechanical patterns. Also, the first simulation cycle was used to ensure that all sequences had the same starting geometry. A side effect is that the generated 

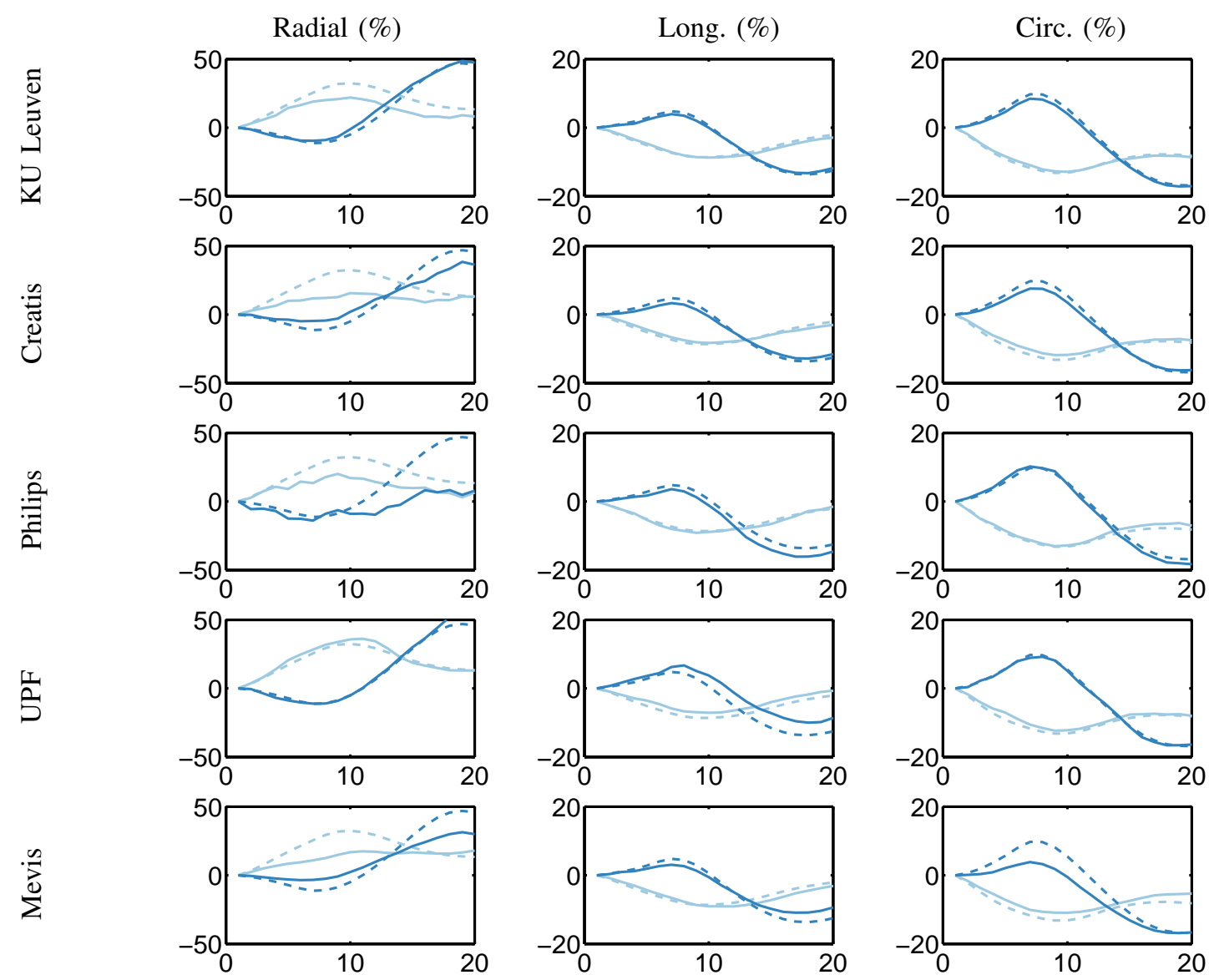

seg. $9--=\operatorname{seg} .9$ (gr. truth)

seg. 12 ---seg. 12 (gr. truth)

Fig. 12. For each methodology and component, strain curves are plotted in two segments: AHA segments \#9 (septal, mid-level) and \#12 (lateral wall,mid-level). Ground truth curves are overlaid in dashed.

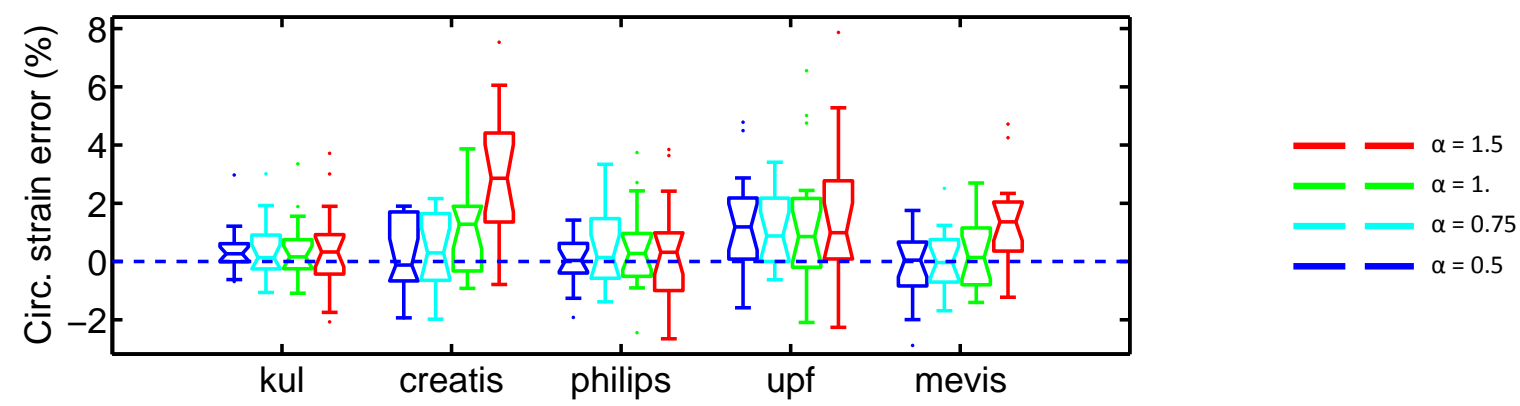

Fig. 13. Sensitivity of circumferential strain to different SNRs between myocardium and blood pool intensities. Difference between true and estimated values are plotted as box plots per methodology for decreasing SNRs. As in Fig. 8, the lowest SNR level is plotted in red. See the legend for intermediate SNR colors and Fig. 5 for the meaning of $\alpha$. 
heart cycle was not perfectly periodic. Finally, other motion patterns should be considered to make the database more representative, including patterns triggered by hypertensive and genetic backgrounds.

As biomechanical and ultrasound simulations technologies are constantly evolving, frequent iterations are required from this starting point. Rather than being shaped as a static publication, an efficient open validation strategy must be implemented on an open platform where both model and tracking methodologies can be frequently updated. One example of such a framework is the recently developed VIP platform [64].

Despite these limitations, all data and obtained tracking results in this paper are opened to the community in the form of supplemental material to this report. Since this study only covers partially the state of the art, developers of motion and strain algorithms from B-mode images are encouraged to apply their methodology to this data for benchmarking with existing technologies. Besides data and tracking results, strain computation code is also made available to ensure that the comparison here presented is fully reproducible. Nonetheless, it should be noted that newly reported results will not have the same constraints as participants to this study. Indeed, all participants to this paper were blind to the ground truth and had strict time constraints (see Sect. IV-F). Note that the data generated in this paper is currently limited to B-mode images. The comparison to RF-based quantification techniques will be addressed in future work. This will allow to assess the gain in accuracy obtained by including phase information within the analysis [3].

Results presented in this paper showed moderate variations in comparison to inter-vendor comparisons performed in 3D [5] or 2D [65]. A possible explanation is that we enforced several elements of the tracking-based pipeline to be consistent between methodologies. We used the same strain computation method, projected the strain tensor on the same anatomical system of coordinates and used the same segmentation and AHA segments definitions. The strain computation used in this paper did not introduce any additional smoothing. All regularization parameters were adjusted by each participant optimizing tracking accuracy on the normal sequence at highest SNR. Hence, evaluating separately the impact of tracking and regularization strategies on strain accuracy must be addressed in future work.

\section{CONCLUSION}

This paper introduced an advanced pipeline for generating a synthetic validation database for 3D US strain quantification algorithms. The focus was set on modeling two typical challenges for 3D deformation imaging. The first challenge is the detection of abnormal myocardium segments with reduced cardiac function, as in the case of acute ischemy. The second challenge is the detection of dyssynchrony, as resulting from conduction problems such as LBBB.

Five tracking methodologies were applied to the generated synthetic data and compared in this study. Accuracy levels were found to be similar between methodologies. Dyssynchrony datasets gave a lower accuracy, indicating that dilated geometries represent the biggest challenge for the evaluated algorithms. The inclusion of an intense pericardial layer around the myocardium had no significant impact on tracking accuracy. Overall, the method provided by KU Leuven had the highest displacement accuracy.

Regarding strain, it was observed that all methods (but UPF) tended to underestimate radial strain. Longitudinal strain had low values in our simulations that were difficult to recover by the tracking algorithms. Circumferential strain however was accurately quantified and all algorithms appeared to be able of discriminating diseased from healthy segments on this dataset.

Robustness to low contrasts between tissue and blood pool had a different impact depending on the methodology. As the tracking of image borders became more difficult at low SNRs, both tracking and strain accuracies were affected. For the range of evaluated SNRs, tracking accuracy remained in the order of 1-1.5 $\mathrm{mm}$ and median circumferential error was smaller than $3 \%$.

The data is made publicly available (http://bit.ly/3dstraus) to ensure that other teams can compare their result to this initial benchmark. Further research will be dedicated to improving the realism of the mechanical and imaging simulations. From the mechanical side of view, more motion patterns will be modeled and boundary conditions will be adjusted to improve the realism of longitudinal strain and torsion values. For the ultrasound part, imaging artifacts will be learned from real images to correctly render typical clutter, valve and pericardium artifacts.

\section{REFERENCES}

[1] G. R. Sutherland, G. Di Salvo, P. Claus, J. D’hooge, and B. Bijnens, "Strain and strain rate imaging: a new clinical approach to quantifying regional myocardial function," J Am Soc Echocardiogr, vol. 17, no. 7, pp. 788-802, 2004.

[2] J.-U. Voigt and F. Flachskampf, "Strain and strain rate," Z Kardiol, vol. 93, no. 4, pp. 249-258, 2004.

[3] R. G. Lopata, M. M. Nillesen, J. M. Thijssen, L. Kapusta, and C. L. de Korte, "Three-dimensional cardiac strain imaging in healthy children using RF-data," Ultrasound Med Biol, vol. 37, no. 9, pp. 1399 - 1408, 2011.

[4] E. Konofagou and J. Ophir, "A new elastographic method for estimation and imaging of lateral displacements, lateral strains, corrected axial strains and poissons ratios in tissues," Ultrasound Med Biol, vol. 24 no. 8, pp. 1183-1199, 1998.

[5] E. Gayat, H. Ahmad, L. Weinert, R. Lang, and V. Mor-Avi, "Reproducibility and inter-vendor variability of left ventricular deformation measurements by three-dimensional speckle-tracking echocardiography," J Am Soc Echocardiogr, vol. 24, no. 8, pp. 878-885, 2011.

[6] T. H. Marwick, "Will standardization make strain a standard measurement?" J Am Soc Echocardiogr, vol. 25, no. 11, pp. 1204-1206, Nov. 2012.

[7] L. de Isla, D. Balcones, C. Fernández-Golfín, P. Marcos-Alberca, C. Almería, J. Rodrigo, C. Macaya, and J. Zamorano, "Threedimensional-wall motion tracking: a new and faster tool for myocardial strain assessment: comparison with two-dimensional-wall motion tracking," J Am Soc Echocardiogr, vol. 22, no. 4, pp. 325-330, 2009.

[8] P. Reant, L. Barbot, C. Touche, M. Dijos, F. Arsac, X. Pillois, M. Landelle, R. Roudaut, and S. Lafitte, "Evaluation of global left ventricular systolic function using three-dimensional echocardiography speckletracking strain parameters," J Am Soc Echocardiogr, vol. 25, no. 1, pp. 68-79, 2011.

[9] F. Maffessanti, H.-J. Nesser, L. Weinert, R. Steringer-Mascherbauer, J. Niel, W. Gorissen, L. Sugeng, R. M. Lang, and V. Mor-Avi, "Quantitative evaluation of regional left ventricular function using three-dimensional speckle tracking echocardiography in patients with and without heart disease," Am J Cardiol, vol. 104, no. 12, pp. 1755-1762, Dec. 2009. 
[10] K. Saito, H. Okura, N. Watanabe, A. Hayashida, K. Obase, K. Imai, T. Maehama, T. Kawamoto, Y. Neishi, and K. Yoshida, "Comprehensive evaluation of left ventricular strain using speckle tracking echocardiography in normal adults: Comparison of threedimensional and two-dimensional approaches," J Am Soc Echocardiogr, vol. 22, no. 9, pp. 1025-1030, Sep. 2009.

[11] R. Jasaityte, B. Heyde, V. Ferferieva, B. Amundsen, D. Barbosa, D. Loeckx, G. Kiss, F. Orderud, P. Claus, H. Torp, and J. Dhooge, "Comparison of a new methodology for the assessment of 3D myocardial strain from volumetric ultrasound with 2D speckle tracking," Int J Card Imaging, vol. 28, pp. 1049-1060, 2012.

[12] B. Amundsen, T. Helle-Valle, T. Edvardsen, H. Torp, J. Crosby, E. Lyseggen, A. Støylen, H. Ihlen, J. Lima, O. Smiseth et al., "Noninvasive myocardial strain measurement by speckle tracking echocardiography: validation against sonomicrometry and tagged magnetic resonance imaging," J Am Coll Cardiol, vol. 47, no. 4, pp. 789-793, 2006.

[13] A.-C. Pouleur, J.-B. le Polain de Waroux, A. Pasquet, B. L. Gerber, O. Gérard, P. Allain, and J.-L. J. Vanoverschelde, "Assessment of left ventricular mass and volumes by three-dimensional echocardiography in patients with or without wall motion abnormalities: comparison against cine magnetic resonance imaging," Heart, vol. 94, no. 8, pp. 1050-1057, 2008.

[14] J. Jensen and N. Svendsen, "Calculation of pressure fields from arbitrarily shaped, apodized, and excited ultrasound transducers," IEEE Trans. Ultrason., Ferroelectr., Freq. Control, vol. 39, no. 2, pp. 262-267, 1992.

[15] H. Gao, H. Choi, P. Claus, S. Boonen, S. Jaecques, G. Van Lenthe, G. Van Der Perre, W. Lauriks, and J. D'hooge, "A fast convolutionbased methodology to simulate 2-D/3-D cardiac ultrasound images," IEEE Trans. Ultrason., Ferroelectr., Freq. Control, vol. 56, no. 2, pp. 404-409, 2009.

[16] A. Elen, H. Choi, D. Loeckx, H. Gao, P. Claus, P. Suetens, F. Maes, and J. D'hooge, "Three-dimensional cardiac strain estimation using spatiotemporal elastic registration of ultrasound images: A feasibility study," IEEE Trans. Med. Imag., vol. 27, no. 11, pp. 1580-1591, 2008.

[17] Q. Duan, P. Moireau, E. Angelini, D. Chapelle, and A. Laine, "Simulation of 3D ultrasound with a realistic electro-mechanical model of the heart," FIMH, vol. 4466, pp. 463-473, 2007.

[18] M. De Craene, P. Allain, H. Gao, A. Prakosa, S. Marchesseau, L. Hilpert, O. Somphone, H. Delingette, S. Makram-Ebeid, N. Villain, J. D’hooge, M. Sermesant, and E. Saloux, "Synthetic and phantom setups for the second cardiac motion analysis challenge (cMAC2)," in STACOM, ser. LNCS, vol. 7746. Springer, 2012, pp. 125-133.

[19] B. Lesniak Plewinska, S. Cygan, K. Kaluzynski, J. D’hooge, J. Zmigrodzki, E. Kowalik, M. Kordybach, and M. Kowalski, "A dual-chamber, thick-walled cardiac phantom for use in cardiac motion and deformation imaging by ultrasound," Ultrasound Med Biol, vol. 36, no. 7, pp. 11451156,2010

[20] K. J. M. Surry, H. J. B. Austin, A. Fenster, and T. M. Peters, "Poly(vinyl alcohol) cryogel phantoms for use in ultrasound and MR imaging," Phys Med Biol, vol. 49, no. 24, p. 5529, 2004.

[21] B. Heyde, S. Cygan, H. Choi, B. Lesniak-Plewinska, D. Barbosa A. Elen, P. Claus, D. Loeckx, K. Kaluzynski, and J. D'hooge, "Regional cardiac motion and strain estimation in three-dimensional echocardiography: a validation study in thick-walled univentricular phantoms," IEEE Trans. Ultrason., Ferroelectr., Freq. Control, vol. 59, no. 4, pp. 668-682, 2012.

[22] M. Ashraf, A. Myronenko, T. Nguyen, A. Inage, W. Smith, R. Lowe, K. Thiele, C. Kroeker, J. Tyberg, J. Smallhorn et al., "Defining left ventricular apex-to-base twist mechanics computed from high-resolution 3D echocardiography: Validation against sonomicrometry," JACC: Cardiovascular Imaging, vol. 3, no. 3, pp. 227-234, 2010.

[23] Y. Seo, T. Ishizu, Y. Enomoto, H. Sugimori, and K. Aonuma, "Endocardial surface area tracking for assessment of regional LV wall deformation with 3D speckle tracking imaging," JACC: Cardiovascular Imaging, vol. 4, no. 4, pp. 358-365, 2011.

[24] Y. Seo, T. Ishizu, Y. Enomoto, H. Sugimori, M. Yamamoto, T. Machino, R. Kawamura, and K. Aonuma, "Validation of 3-dimensional speckle tracking imaging to quantify regional myocardial deformation / clinical perspective," Circulation: Cardiovascular Imaging, vol. 2, no. 6, pp. 451-459, 2009.

[25] B. Heyde, S. Bouchez, S. Thieren, M. Vandenheuvel, R. Jasaityte, D. Barbosa, P. Claus, F. Maes, P. Wouters, and J. Dhooge, "Elastic image registration to quantify $3 \mathrm{~d}$ regional myocardial deformation from volumetric ultrasound: Experimental validation in an animal model," Ultrasound Med Biol, 2013, accepted to appear.

[26] R. Jasaityte, B. Heyde, and J. D'hooge, "Current state of three- dimensional myocardial strain estimation using echocardiography," $J$ Am Soc Echocardiogr, vol. 26, no. 1, pp. 15-28, Jan. 2013.

[27] A. Prakosa, K. Mcleod, M. Sermesant, and X. Pennec, "Evaluation of ilogdemons algorithm for cardiac motion tracking in synthetic ultrasound sequence," in STACOM, ser. LNCS, vol. 7746. Springer, 2013, pp. 178187.

[28] B. Heyde, D. Barbosa, P. Claus, F. Maes, and J. D'hooge, "Threedimensional cardiac motion estimation based on non-rigid image registration using a novel transformation model adapted to the heart," in STACOM, ser. LNCS, vol. 7746. Springer, 2013, pp. 142-150.

[29] L. Tautz, A. Hennemuth, and H.-O. Peitgen, "Quadrature filter based analysis of myocardial deformation based on 3D ultrasound," in STACOM, ser. LNCS, vol. 7746. Springer, 2013, pp. 169-177.

[30] G. Piella, A. R. Porras, M. De Craene, N. Duchateau, and A. F. Frangi, "Temporal diffeomorphic free form deformation to quantify changes induced by left and right bundle branch block and pacing," in STACOM, ser. LNCS, vol. 7746. Springer, 2013, pp. 134-141.

[31] M. Alessandrini, H. Liebgott, D. Barbosa, and O. Bernard, "Monogenic phase based optical flow computation for myocardial motion analysis in 3D echocardiography," in STACOM, ser. LNCS, vol. 7746. Springer, 2013, pp. 159-168.

[32] O. Somphone, C. Dufour, L. Hilpert, S. Makram Ebeid, N. Villain, M. De Craene, P. Allain, and E. Saloux, "Motion estimation in 3D echocardiography using smooth field registration," in STACOM, ser. LNCS, vol. 7746. Springer, 2013, pp. 151-158.

[33] Y. Wang, B. Georgescu, H. Houle, and D. Comaniciu, "Volumetric myocardial mechanics from $3 \mathrm{D}+\mathrm{t}$ ultrasound data with multi-model tracking," in STACOM, ser. LNCS, vol. 6364. Springer, 2010, pp. 184-193.

[34] K. Leung, M. Danilouchkine, M. van Stralen, N. de Jong, A. van der Steen, and J. Bosch, "Left ventricular border tracking using cardiac motion models and optical flow," Ultrasound Med Biol, vol. 37, no. 4, pp. 605-616, 2011.

[35] X. Papademetris, A. Sinusas, D. Dione, J. Duncan et al., "Estimation of 3D left ventricular deformation from echocardiography." Med Image Anal, vol. 5, no. 1, p. 17, 2001.

[36] X. Papademetris, A. Sinusas, D. Dione, R. Constable, and J. Duncan, "Estimation of 3D left ventricular deformation from medical images using biomechanical models," IEEE Trans. Med. Imag., vol. 21, no. 7, pp. 786-800, 2002.

[37] M. Sermesant, Y. Coudière, H. Delingette, N. Ayache, and J.-A. Désidéri, "An electro-mechanical model of the heart for cardiac image analysis," in Medical Image Computing and Computer-Assisted Intervention-MICCAI 2001. Springer, 2001, pp. 224-231.

[38] A. Myronenko, X. Song, and D. Sahn, "Maximum likelihood motion estimation in 3D echocardiography through non-rigid registration in spherical coordinates," FIMH, pp. 427-436, 2009.

[39] M. De Craene, G. Piella, O. Camara, N. Duchateau, E. Silva, A. Doltra J. D'hooge, J. Brugada, M. Sitges, and A. F. Frangi, "Temporal diffeomorphic free-form deformation: Application to motion and strain estimation from 3D echocardiography." Med Image Anal, vol. 16, no. 2, pp. 427-450, Feb 2012.

[40] Q. Duan, E. Angelini, O. Gerard, S. Homma, and A. Laine, "Comparing optical-flow based methods for quantification of myocardial deformations on RT3D ultrasound,' in ISBI. IEEE, 2006, pp. 173-176.

[41] T. Mansi, X. Pennec, M. Sermesant, H. Delingette, and N. Ayache, "iLogDemons: A demons-based registration algorithm for tracking incompressible elastic biological tissues," Int. J. of Computer Vision, vol. 92, no. 1, pp. 92 - 111, 2011.

[42] H. Knutsson and M. Andersson, "Morphons: Segmentation using elastic canvas and paint on priors," in Symp Image Anal SSBA, 2005, pp. 73-76.

[43] E. Angelini and O. Gerard, "Review of myocardial motion estimation methods from optical flow tracking on ultrasound data," in EMBS. IEEE, 2006, pp. 1537-1540.

[44] M. Lubinski, S. Emelianov, and M. O’Donnell, "Speckle tracking methods for ultrasonic elasticity imaging using short-time correlation," IEEE Trans. Ultrason., Ferroelectr., Freq. Control, vol. 46, no. 1, pp. 82-96, 1999.

[45] J. Crosby, B. Amundsen, T. Hergum, E. Remme, S. Langeland, and H. Torp, "3D speckle tracking for assessment of regional left ventricular function," Ultrasound Med Biol, vol. 35, no. 3, pp. 458-471, 2009.

[46] M. Ledesma-Carbayo, J. Kybic, M. Desco, A. Santos, M. Suhling, P. Hunziker, and M. Unser, "Spatio-temporal nonrigid registration for ultrasound cardiac motion estimation," IEEE Trans. Med. Imag., vol. 24, no. 9, pp. 1113-1126, 2005. 
[47] L. Tautz, A. Hennemuth, and H.-O. Peitgen, "Motion analysis with quadrature filter based registration of tagged MRI sequences," in STACOM, ser. LNCS, vol. 7085. Springer, 2012, pp. 78-87.

[48] C. Tobon-Gomez, M. D. Craene, K. McLeod, L. Tautz, W. Shi, A. Hennemuth, A. Prakosa, H. Wang, G. Carr-White, S. Kapetanakis, A. Lutz, V. Rasche, T. Schaeffter, C. Butakoff, O. Friman, T. Mansi, M. Sermesant, X. Zhuang, S. Ourselin, H.-O. Peitgen, X. Pennec, R. Razavi, D. Rueckert, A. Frangi, and K. Rhode, "Benchmarking framework for myocardial tracking and deformation algorithms: An open access database," Medical Image Analysis, 2013, accepted to appear.

[49] O. Ecabert, J. Peters, H. Schramm, C. Lorenz, J. von Berg, M. Walker, M. Vembar, M. Olszewski, K. Subramanyan, G. Lavi, and J. Weese, "Automatic model-based segmentation of the heart in CT images," IEEE Trans. Med. Imag., vol. 27, no. 9, pp. 1189 -1201, sept. 2008.

[50] D. Chapelle, P. Le Tallec, P. Moireau, and M. Sorine, "An energypreserving muscle tissue model: formulation and compatible discretizations," Int J Multiscale Com, vol. 10, no. 2, pp. 189-211, 2012.

[51] S. Marchesseau, H. Delingette, M. Sermesant, and N. Ayache, "Fast parameter calibration of a cardiac electromechanical model from medical images based on the unscented transform," Biomech Model Mechanobiol, pp. 1-17, 2012.

[52] M. Sermesant, R. Chabiniok, P. Chinchapatnam, T. Mansi, F. Billet, P. Moireau, J. Peyrat, K. Wong, J. Relan, K. Rhode, M. Ginks, P. Lambiase, H. Delingette, M. Sorine, C. Rinaldi, D. Chapelle, R. Razavi, and N. Ayache, "Patient-specific electromechanical models of the heart for the prediction of pacing acute effects in CRT: A preliminary clinical validation," Med Image Anal, vol. 16, no. 1, pp. 201-215, 2012.

[53] S. Marchesseau, H. Delingette, M. Sermesant, M. Sorine, K. Rhode, S. Duckett, C. Rinaldi, R. Razavi, and N. Ayache, "Preliminary specificity study of the Bestel-Clement-Sorine electromechanical model of the heart using parameter calibration from medical images," $J$ Mech Behav Biomed Mater, vol. 20, pp. 259-271, 2013.

[54] AHA Writing Group on Myocardial Segmentation and Registration for Cardiac Imaging, M. D. Cerqueira, N. J. Weissman, V. Dilsizian, A. K. Jacobs, S. Kaul, W. K. Laskey, D. J. Pennell, J. A. Rumberger, T. Ryan, and M. S. Verani, "Standardized myocardial segmentation and nomenclature for tomographic imaging of the heart: A statement for healthcare professionals from the cardiac imaging committee of the council on clinical cardiology of the american heart association," Circulation, vol. 105, no. 4, pp. 539-542, 2002.

[55] O. Somphone, M. D. Craene, R. Ardon, B. Mory, P. Allain, H. Gao, J. Dhooge, S. Marchesseau, M. Sermesant, and E. Saloux, "Fast myocardial motion and strain estimation in 3D cardiac ultrasound with sparse demons," in ISBI, IEEE, Ed., 2013, pp. 1170-1173.

[56] H. Gao, T. Hergum, H. Torp, and J. Dhooge, "Comparison of the performance of different tools for fast simulation of ultrasound data," Ultrasonics, vol. 52, no. 5, pp. 573 - 577, 2012.

[57] M. Alessandrini, A. Basarab, H. Liebgott, and O. Bernard, "Myocardial motion estimation from medical images using the monogenic signal," IEEE Trans. Image Process., vol. 22, no. 3, pp. 1084-1095, 2012.

[58] D. Rueckert, L. Sonoda, C. Hayes, D. Hill, M. Leach, and D. Hawkes, "Nonrigid registration using free-form deformations: Application to breast MR images," IEEE Trans. Med. Imag., pp. 712-721, 1999.

[59] C. Petitjean, N. Rougon, and P. Cluzel, "Assessment of myocardial function: a review of quantification methods and results using tagged MRI," J Cardiovasc Magn Reson, vol. 7, no. 2, pp. 501-516, 2005.

[60] M. Alessandrini, H. Liebgott, D. Friboulet, and O. Bernard, "Simulation of realistic echocardiographic sequences for ground truth validation of motion estimation," in ICIP, Orlando, USA, 2012, pp. 2329-2332.

[61] A. Prakosa, M. Sermesant, H. Delingette, S. Marchesseau, E. Saloux, P. Allain, N. Villain, and N. Ayache, "Generation of synthetic but visually realistic time series of cardiac images combining a biophysical model and clinical images," IEEE Trans. Med. Imag., vol. 32, no. 1, pp. 99-109, 2012.

[62] F. Varray, C. Cachard, A. Ramalli, P. Tortoli, and O. Basset, "Simulation of ultrasound nonlinear propagation on gpu using a generalized angular spectrum method," EURASIP J Image Video Process, vol. 2011, no. 17, pp. 1-6, 2011.

[63] T. Yingchoncharoen, S. Agarwal, Z. Popović, and T. Marwick, "Normal ranges of left ventricular strain: A meta-analysis," J Am Soc Echocardiogr, vol. 26, no. 2, pp. 185-91, 2012.

[64] T. Glatard, C. Lartizien, B. Gibaud, R. da Silva, G. Forestier, F. Cervenansky, M. Alessandrini, H. Benoit-Cattin, O. Bernard, S. CamarasuPop et al., "A virtual imaging platform for multi-modality medical image simulation," IEEE Trans. Med. Imag., vol. 32, no. 1, pp. 110-118, 2012.
[65] N. Risum, S. Ali, N. T. Olsen, C. Jons, M. G. Khouri, T. K. Lauridsen, Z. Samad, E. J. Velazquez, P. Sogaard, and J. Kisslo, "Variability of global left ventricular deformation analysis using vendor dependent and independent two-dimensional speckle-tracking software in adults," J Am Soc Echocardiogr, vol. 25, no. 11, pp. 1195 - 1203, 2012. 